\title{
Slow Conduction Regions as a Valuable Vectorcardiographic Parameter for the Non-Invasive Identification of Atrial Flutter Types
}

\author{
Samuel Ruipérez-Campillo ${ }^{1,3}$, Sergio Castrejón ${ }^{2}$, Marcel Martínez ${ }^{2}$, Raquel Cervigón ${ }^{4}$, \\ Olivier Meste ${ }^{5}$, José Luis Merino ${ }^{2}$, José Millet ${ }^{1}$, Francisco Castells ${ }^{1}$ \\ ${ }^{1}$ Bio-ITACA Research Group, ITACA Institute, Universitat Politècnica de València, Valencia, Spain \\ ${ }^{2}$ Unidad de Arritmias y Electrofisiología Robotizada, Hospital Universitario La Paz, Madrid, Spain \\ ${ }^{3}$ Bioengineering and Aerospace Engineering Department, Universidad Carlos III, Madrid, Spain \\ ${ }^{4}$ Universidad de Castilla la Mancha, Cuenca, Spain \\ ${ }^{5}$ Université Cote d'Azur, Nice, France
}

\begin{abstract}
The objective of this study is to non-invasively characterise a variety of atrial flutter (AFL) types, defined by a maroreentrant circuit. A vectorcardiographic approach is proposed to compare atrial macroreentrant circuits. Vectorcardiogram (VCG) arechetypes are computed so that parameters such as similarity among loops can be calculated. The methodology was employed in a set of artificial VCGs created from a computational simulation based on a mathematical model and in signals from real patients. Adenosine was used to block the ventricular contribution to the ECG signal, later transformed to a VCG analysed from different perspectives. Results demonstrate a high similarity for cases belonging to a group with its archetype in synthetic and real cases. Slow conduction velocity regions were found to be very well represented in VCGs, in accordance with AFL mechanisms and its importance when characterising atrial macroreentries. The conclusion is that our methodology allows differentiation between the most recurrent types of AFL through the analysis of its VCG representation, predicting the presence of slow conduciton regions along the macroreentry. This can be very useful when planning in advance the ablation procedure.
\end{abstract}

\section{Introduction}

The increasing prevalence of AFL [1] and its usual treatment, catheter ablation [2], makes it an important issue to study. The physiological basis of AFL is an atrial macroreentrant circuit [3] sorrounding an obstacle, in either counterclockwise (CCW) or a clockwise $(\mathrm{CW})$ direction. The recreation of the macroreentry is associated to a slow conduction area allowing the full repolarisation of myocites after the refractory period. Depending on where the circuit sustaining the macroreentry of the atria happens, different AFL types arise, being the most prevalent, typical AFL. Recently, electroanatomical mapping systems have eased atrial mapping and ablation. Nevertheless, it requires time and expertise, so new non-invasive mapping techniques such as ECG Imaging are being developed although results may be controversial [4]. Thus, an advantageous information prior to the electrophysiological study would be defining the AFL type and the most likely reentrant circuit from the ECG signal. This would allow simplifying the electroanatomical mapping as the interest area would be known, and would facilitate the procedure, identifying whether it is right or left atrium AFL.

Diagnosis of AFL has barely changed along history, mainly relying on ECG atrial waves, although it is not rare to find false negatives and positives. Furthermore, 2:1 atrio-ventricular ratios are very common, complicating the identification of the repeating T-wave. Some approaches allow cancelling ventricular activity in atrial fibrillation $[5,6]$, but this cannot be applied to AFL as ventricular components are coupled. Neither Blind Source Separation [7] would be sufficient, as three independent atrial components would be needed to compute a VCG loop. Thus, it is still a challenge to doubtlessly retrieve the atrial signal in AFL [8], though administrating adenosine to block the AV node for a short period of time can be a solution.

The vectorcardiographic interpretation is defined as a representation of 3D atrial surface loops. Some parameters have been previously defined to quantify features of the QRS loop [9]. In AFL, VCGs have been described but no clear correlations to physiology were demonstrated [10]. The hypothesis of this study states that there is a correlation between the AFL type and its VCG loop representation, based fundamentally on slow conduction regions. Hence, VCG loops are characterised to determine and differentiate AFL groups. 


\section{Study Design}

To demonstrate the hypothesis, a methodology based on an innovative standpoint of the VCG signal is developed, where slow conduction regions are fundamental. This methodology is tested through computational simulations based on a geometrical model. With this basis, an artificial VCG generator is designed based on a mathematical model. On the other hand, VCGs from patients suffering from AFL are taken from Hospital Universitario la Paz (Madrid, Spain) - at the Robotic Cardiac Electrophysiology and Arrhythmia Unit. This procedures were in accordance with the Helsinki Protocol. AFL characterisation from clinical data was performed by the cardiologists and electrophysiologists, who provided our gold standard. ECG signals were transformed to VCGs to extract features and quantifiable parameters which underwent later statistical tests.

\section{Materials and Methods}

\subsection{Synthetic Data: Mathematical Model}

In order to test the methodology and support the hypothesis, a geometrical model is created to generate loops with similar features to real atrial loops, were slow regions take an essential role. 8 groups are created defined by the location of slow conducting regions, and a wide range of parameters are defined to offer a diverse variability among the synthetic cases belonging to the same group - from plane rotation to complex curvatures.

Synthetic atrial VCG loops are generated from projections of ellipsoidal figures randomised from Euler expression. In addition to semi-slow regions naturally comming from higher density of points when discretising near the minor semi-axis (see figure 1), a characteristic slow region is defined by a non-uniform discretisation as follows:

$$
\Delta \theta_{n}=\beta\left|\cos \left(n \frac{\pi}{N}+\phi\right)\right|+\Delta \theta_{\min },
$$

where $\Delta \theta_{n}$ is the angular increment at each of the $n$ samples, the minimum angular increment is $\Delta \theta_{\min }$, defining the minimum velocity, in contrast to $\beta+\Delta \theta_{\min }$ which defines the maximum angular variation, standing for high velocity regions, $N$ the total number of samples, and $\phi$, the phase argument determining the position of slow regions. In addition, the summation of all angular increments along the entire loop defined by Eq. (1) must equals $2 \pi$.

To reduce the planarity of the path and introduce 3D curvature variations, weighted $\left(P_{1}, P_{2}\right.$ and $\left.P_{3}\right)$ frequency modulating functions $\left(F_{1}, F_{2}\right.$ and $\left.F_{3}\right)$ are introduced in the definition of the radius of the projections, which also depends on the ellipse semi-axes $x$ and $y$ :

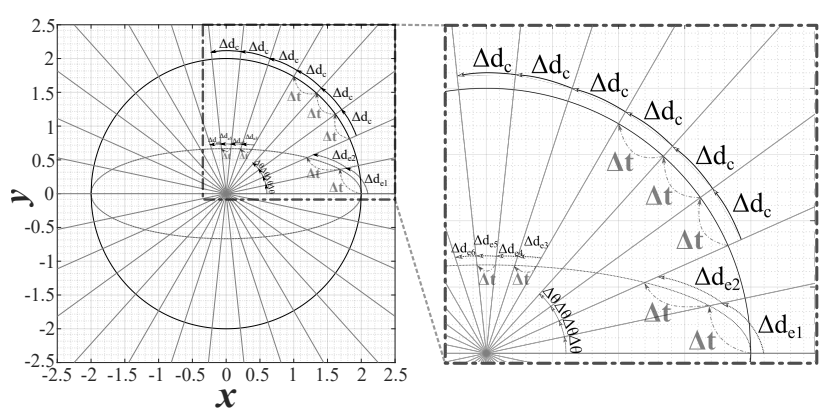

Figure 1: Continuous angular discretisation on a 2D plane over a circle and an ellipse to highlight how concentration of points arise near minor semi-axes of the ellipse, translated as a semi-slow velocity profile when time discretisations are constant.

$$
r(\theta)=\frac{x \cdot y}{\sqrt{\left(x \cdot \cos (\theta)+P_{1} F_{1}\right)^{2}+\left(y \cdot \sin (\theta)+P_{2} F_{2}\right)^{2}+P_{3} F_{3}}},
$$

Sharp peaks may appear in the the velocity profile, so the signal is low-pass filtered with an adaptation of the Savitzky-Golay filter over the three axis (see figure 2 B-C). With the possibility of rotation around the cartesian axes, 4 models are defined depending on the position of the slow conduction region.

Furthermore, two directions (CW and $\mathrm{CCW}$ ) are defined for each type, considering 8 independent groups: 1 to 4 and 5 to $8, \mathrm{CW}$ and $\mathrm{CCW}$ respectively. An example of a synthetic VCG and its velocity profile before and after post-processing is displayed in figure 2 .
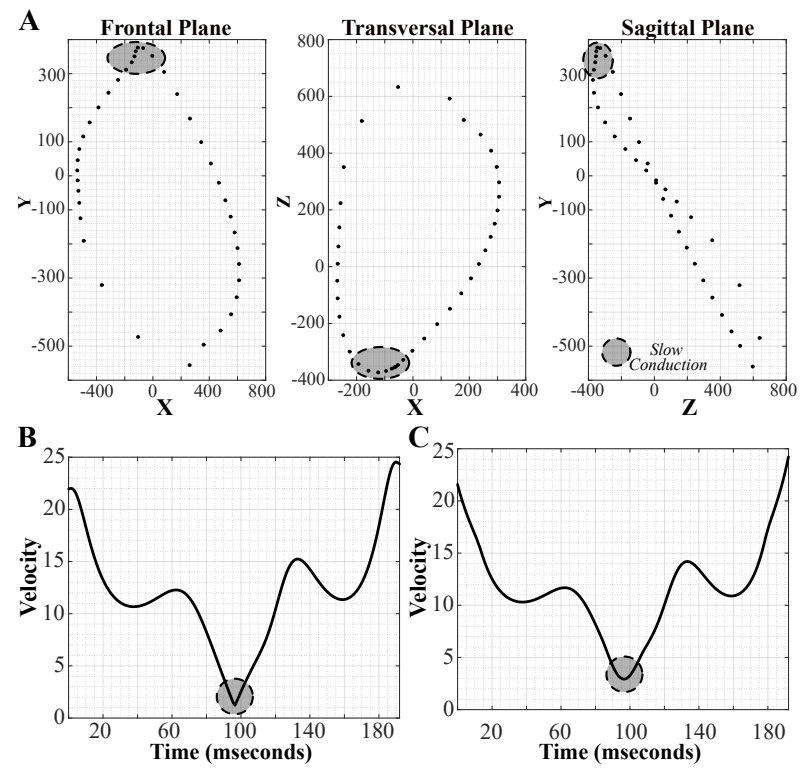

Figure 2: Example of a simulated VCG. A. Frontal, Transversal and Saggital planes. B. Non-filtered velocity Profile. C. Velocity Profile after filtering with implemented adapted Savitzky-Golay. 


\subsection{Clinical Data}

The protocol comprissed 30 patients suffering from AFL. ECG and EGM were registered with $1 \mathrm{kHz}$ of sampling frequency by a polygraph (Labsystem pro, Bard, Boston Scientific), synchronised to data generated by the electronavigator. Adenosine was used during ECG registration, eliminating ventricular contributions. Summarising, the patients analysed belong to the following groups: 5 common CW AFL, 6 common CCW AFL, 3 perimitral CW AFL, 8 perimitral CCW AFL and 8 miscellaneous, belonging to other groups such as pulmonar or cavae periveins.

\subsection{Methods}

\subsubsection{Preprocessing}

Atrial VCG loops are computed from segments of ECG recordings under the effect of adenosine. Signals are filtered between $1 \mathrm{~Hz}$ and $30 \mathrm{~Hz}$ and filtered biderectionally, minimising transient distortion. VCGs were computed through Inverse Dower's Transform, estimating Frank's leads from 12-lead ECG system. A VCG reconstruction is depicted in figure 3. The consistence among the loops is computed to determine their repeatably, and thus, the quality of the signal. From 10 atrial loops, consistence is computed using Eigenvalue Decomposition, being near to the value of 1 when the AFL loop was repeatible.

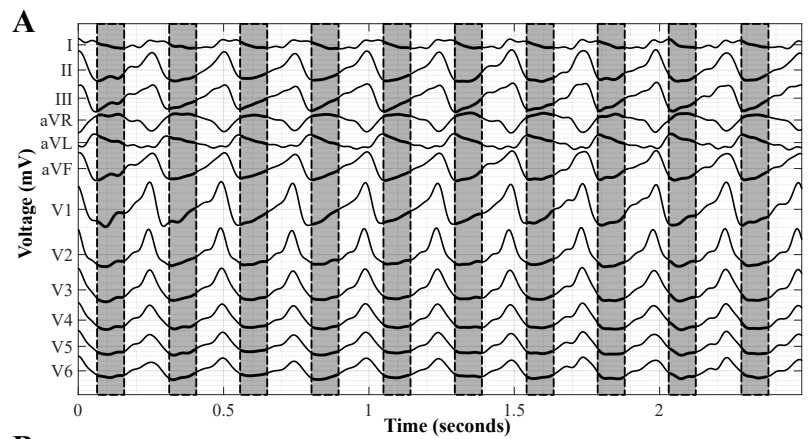

B

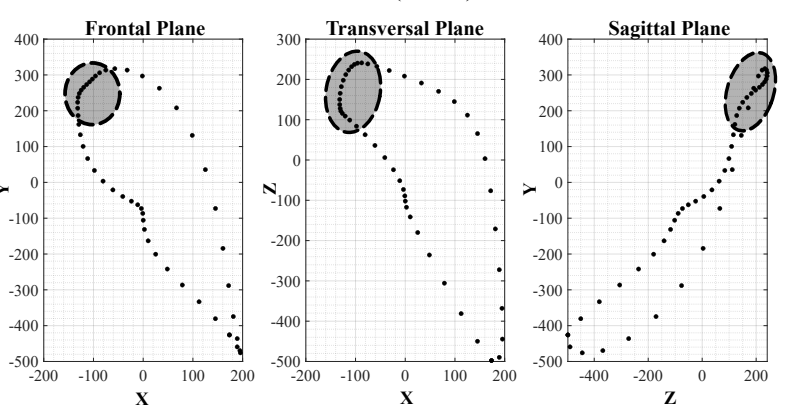

Figure 3: Signal from a patient suffering from perimitral CCW AFL. A. ECG with shaded slow regions. B. VCG reconstruction with slow regions shaded

\subsubsection{VCG Archetypes}

Archetypes for each AFL group are created (in both real and synthetic cases), from the average of normalised VCGs aligned in time using a least squares minimisation approach. Thus, new unknown or non-characterised cases can be compared to these archetypes.

\subsubsection{Characterisation of VCG Loops}

A wide assortment of features are defined to characterise VCG loops. Similarity between VCG loops is computed from the point-by-point correlation as:

$$
S=\frac{1}{N} \sum_{i=1}^{N} \frac{\mathbf{x}_{\mathbf{i}}^{\mathbf{T}} \mathbf{y}_{\mathbf{i}}}{\left\|\mathbf{x}_{\mathbf{i}}\right\|\left\|\mathbf{y}_{\mathbf{i}}\right\|},
$$

where $\mathbf{x}_{\mathbf{i}}$ and $\mathbf{y}_{\mathbf{i}}$ are the 3-dimensional vectors of each VCG loop, at the $i$-th sample and the number of samples is defined by $N$. Note that slow conduction regions represented as cluster of points will provide a higher weight to this parameter based on the directions of the vectors (see figure 3). To identify the most alike archetype, similarity to all of them is computed, having the highest coefficient for the most similar. In clinical cases, archetypes are computed according to the Leave One Out algorithm.

The velocity profile is also assessed as slow velocity regions are important for our hypothesis. The units of the profile is expressed in voltage difference over a temporal scale [V/Cycle]. The following slow conduction regions quantifiable parameters are defined: distance fraction over the macroreentry when the signal travels with slow velocity $\left(S V_{D}\right)$, the temporal fraction over a cycle $\left(S V_{T}\right)$ and their rate $\left(S V_{R}\right)$. Angular velocity $\omega$ can also be computed to better understand the velocity profile, from the $3 \mathrm{D}$ vector $u$ at the $i$-th sample and the sampling period $T_{s}$ :

$$
\omega_{i}=\frac{1}{T_{s}} \arccos \left(\frac{u_{\mathbf{i}}^{\mathbf{T}} u_{\mathbf{i}+\mathbf{1}}}{\left\|u_{\mathbf{i}}\right\|\left\|u_{\mathbf{i}+\mathbf{1}}\right\|}\right)
$$

Another interesting parameter designed is the complexity of the pathway $(C)$, which evaluates the changes in direction along the trajectory. The accumulation of angle variations is lower bounded by $2 \pi$, so it can be normalised as depicted in eq. (5). The simplest possible option leads to $C=0$ while a very complex pathway tends to $C=1$.

$$
C=1-\frac{2 \pi}{\sum_{i} \arccos \left(\frac{\left(\mathbf{x}_{\mathbf{i}}-\mathbf{x}_{\mathbf{i}-1}\right)^{T}\left(\mathbf{x}_{\mathbf{i}+1}-\mathbf{x}_{\mathbf{i}}\right)}{\left\|\mathbf{x}_{\mathbf{i}}-\mathbf{x}_{\mathbf{i}-\mathbf{1}}\right\|\left\|\mathbf{x}_{\mathbf{i}+\mathbf{1}}-\mathbf{x}_{\mathbf{i}}\right\|}\right)}
$$

\subsubsection{Statistical Analysis}

The synthetic cases were analysed using the ANOVA test, following Snedecor's F distribution. The null hypothesis is rejected for $p$-value $<0.05$. In this case, Bonferroni's 
correction for multiple comparisons was used. Also, ROC curves were computed comparing each group with the others, quantified through the area under the curve (AUC). For real data, the Kruskal-Wallis test was applied, with the same condition to reject the null hypothesis.

\section{Results}

For the synthetic data all cases showed statistical significance when comparing to the archetype of its group: from $F(3,3996)=959.20, p<0.001$ to $F(3,3996)=$ $1456.28, p<0.001$. Also when performing multiple comparisons in terms of correlation, Bonferroni's test showed high statistical significance for all cases $(p<0.001)$. AUC ranged from 0.895 to 0.923 . Over a controlled environment, frequency modulating functions were tested making $C$ range from 0 to 0.8 . For similarity, slow regions were rotated over both semi-axis, showing a decrease to $S=0.8$ when slow rotating over the slow region and to $S=0.3$ when slow regions were affected by the rotation.

For real data, intra-patient consistence was evaluated showing an average of $0.95 \pm 0.04$. In terms of the similarity between the patients and the archetypes (following the LOO strategy), no values under 0.85 were found for the archetype of their group, and standard deviations were under 0.05 for those. Results are summarised in table 1 .

\begin{tabular}{|l|c|c|c|c|c|}
\cline { 2 - 6 } \multicolumn{1}{c|}{} & $\begin{array}{c}\text { C.CCW } \\
\text { Patients }\end{array}$ & $\begin{array}{c}\text { C.CW } \\
\text { Patients }\end{array}$ & $\begin{array}{c}\text { P.CCW } \\
\text { Patients }\end{array}$ & $\begin{array}{c}\text { P.CW } \\
\text { Patients }\end{array}$ & $\begin{array}{c}\text { Misc. } \\
\text { Patients }\end{array}$ \\
\hline C.CCW Arch. & $\mathbf{0 . 8 5} \pm \mathbf{0 . 0 3}$ & $0.67 \pm 0.11$ & $0.77 \pm 0.05$ & $0.73 \pm 0.12$ & $0.68 \pm 0.14$ \\
\hline C.CW Arch. & $0.68 \pm 0.05$ & $\mathbf{0 . 9 5} \pm \mathbf{0 . 0 3}$ & $0.66 \pm 0.13$ & $0.75 \pm 0.04$ & $0.58 \pm 0.13$ \\
\hline P.CCW Arch. & $0.78 \pm 0.09$ & $0.63 \pm 0.11$ & $\mathbf{0 . 8 7} \pm \mathbf{0 . 0 4}$ & $0.68 \pm 0.12$ & $0.62 \pm 0.18$ \\
\hline P.CW Arch. & $0.76 \pm 0.08$ & $0.75 \pm 0.01$ & $0.70 \pm 0.12$ & $\mathbf{0 . 9 1} \pm \mathbf{0 . 0 2}$ & $0.65 \pm 0.17$ \\
\hline \hline p-value & 0.014 & $<0.01$ & $<0.01$ & 0.62 & N.A. \\
\hline
\end{tabular}

Table 1: Correlation of Patients form all AFL groups to Archetypes

The analysis of velocity profiles using the KruskalWallis test showed statistical significance for $S V_{T}(p=$ $0.047)$, but the null hypothesis was not rejected for $S V_{D}$ $(p=0.146)$ or $S V_{R}(p=0.658)$. For the complexity parameter, higher coefficients are found for perimitral cases, as well as longer periods. The miscellaneous groups including patients with anatomical malformations shows a higher complexity parameter. Furthermore, when performing the Kruskal-Wallis test, statistical significance is reached for the complexity parameter $(p=0.035)$.

\section{Discussion and Conclusions}

AFL is caused by macroreentrant atrial circuits that need a variety of ablation procedures. Currently it is not possible to identify the AFL type before the intervention takes place so in this study VCGs are the grounds of a methodology to find useful information and characterise AFL prior to the ablation approach. The mathematical model sup- ports the hypothesis and highlights the importance of slow regions when characterising AFL. This discrimination capacity is demonstrated with the high AUC values. In the real cases, the highest correlations were found when comparing to the archetype of their own group, with the lowest standard deviation, depicting a promising capacity to identify the AFL when a new case arises. Furthermore, slow regions are consistently found in the same areas for all cases from the same AFL type. Hence, this study proposes a non-invasive approach that helps to characterise AFL from ECG recordings, providing valuable information to plan and manage medical interventions more efficiently.

\section{References}

[1] Herzog E, Argulian E, Levy SB, Aziz EF. Pathway for the management of atrial fibrillation and atrial flutter. Critical pathways in cardiology 2017;16(2):47-52.

[2] Markowitz SM, Thomas G, Liu CF, Cheung JW, Ip JE, Lerman BB. Approach to catheter ablation of left atrial flutters. Journal of Cardiovascular Electrophysiology 2019; 30(12):3057-3067.

[3] Cosío FG, Pastor A, Núñez A, Magalhaes AP, Awamleh P. Atrial flutter: an update. Revista Espanola de Cardiologia English Edition 2006;59(8):816-831.

[4] Rudy Y. Electrocardiographic imaging (ecgi): a new noninvasive imaging modality for cardiac electrophysiology and arrhythmia. In Medical Imaging 2006: Physiology, Function, and Structure from Medical Images, volume 6143. International Society for Optics and Photonics, 2006; 614306.

[5] Castells F, Mora C, Rieta J, Millet J. Estimation of atrial fibrillatory wave from single-lead atrial fibrillation electrocardiograms using principal component analysis concepts. Medical Biological Eng Computing 2005;43:557-560.

[6] Stridh M, Sornmo L. Spatiotemporal qrst cancellation techniques for analysis of atrial fibrillation. IEEE Transactions on Biomedical Eng 2001;48(1):105-111.

[7] Castells F, Rieta JJ, Millet J, Zarzoso V. Spatiotemporal blind source separation approach to atrial activity estimation in atrial tachyarrhythmias. IEEE Transactions on Biomedical Eng 2005;52(2):258-267.

[8] Jacquemet V, Dube B, Nadeau R, LeBlanc AR, Sturmer M, Becker G, Kus T, Vinet A. Extraction and analysis of $\mathrm{t}$ waves in electrocardiograms during atrial flutter. IEEE Transactions on Biomedical Eng 2010;58(4):1104-1112.

[9] Choudhuri S, Ghosal T, Goswami DP, Sengupta A. Planarity of the spatial qrs loop of vectorcardiogram is a crucial diagnostic and prognostic parameter in acute myocardial infarction. Medical hypotheses 2019;130:109-251.

[10] Ng J, Sahakian AV, Fisher W, Swiryn S. A vectorcardiographic approach to understanding the 12-lead electrocardiogram of atrial flutter. In Computers in Cardiology, 2004. IEEE, 2004; 629-632.

Adress of Correspondence: Samuel Ruipérez-Campillo; samuel.ruiperez@alumnos.uc3m.es ITACA Institute Edificio 8G, Acceso B, 1a planta, Universitat Politècnica de Valencia, Camino de Vera S/N 46022 Valencia España +34 963877000 ext 76043 (or +34 678875708) 